\title{
TDEM for Martian in situ resource prospecting missions
}

Roberto Filippini, Cinthya Ottonello, Sergio Pagnan and Giorgio Tacconi

Istituto di Studi sui Sistemi Intelligenti per l'Automazione, CNR, Sezione di Genova, Italy

\begin{abstract}
This paper presents a TDEM (Time Domain Electromagnetic Methods) application, addressed to the search for water on Mars. In this context, the opportunities for a TDEM system as payload in a future mission are investigated for different in situ exploration scenarios. The TDEM sounding capability is evaluated with respect to the expected Martian environment, and some considerations are made about the many unknown variables (above all the background EM noise and the subsoil composition) altogether with the limited resources availability (mission constraints in mass, time and power) and the way they could represent an obstacle for operations and measurements.
\end{abstract}

Key words TDEM - in situ remote sensing - planetary exploration

\section{Introduction}

The large amount of data and images recorded from Mars has given a detailed knowledge of planet environment (surface and atmosphere) thus permitting the scientific community to seek matters of fundamental scientific relevance. First in order of importance, research into any elementary (past or present) form of life on the planet. Since life is mainly correlated to the existence of water, whatever its physical state (liquid, ice, permafrost, other...), the scientific efforts have been turned towards the study of new methodologies that could better support the search for water. Above all, the methodologies deployed for remote sensing observations by means of HF (High Frequency) radars, and high

Mailing address: Dr. Sergio Pagnan, Istituto di Studi sui Sistemi Intelligenti per l'Automazione, CNR, Sezione di Genova, Via De Marini 6, «Torre di Francia», 16149 Genova, Italy; e-mail: pagnan@ian.ge.cnr.it resolution cameras aboard satellites orbiting around the planet (e.g., the Mars Global Surveyor-MGS). Secondly, the in situ exploration of the surface and subsurface.

The study concerns the analysis of a TDEM (Time Domain Electromagnetic Method) system for in situ martian geophysical exploration. TDEM is a controlled source time domain method operating at LF (Low Frequency) regime of propagation. It is able to discriminate the geologically layered structure and detect the signature of water by means of subsoil conductivity estimation (as a function of depth). The TDEM employment, for an in situ survey on Mars, is advisable for many reasons: it is fully controllable in position and strength, it can undergo notable variations due to water presence and the instrument does not need to be in contact with the ground, thus respecting the mission planetary protection and cross contamination requirements.

TDEM (originally developed for detecting massive sulphide deposits) is beginning to emerge due to its high sensitivity that has the best potential for deep hydro-geophysical mapping. This makes it preferable to other electromagnetic methods such as IP (Induced Polar- 
ization), VES (Vertical Electric Sounding), and MT (MagnetoTelluric) that could in principle accomplish the same task.

The purpose of the paper is to investigate TDEM performances when employed in the Martian environment and also to investigate the opportunities for a TDEM system to be housed as scientific payload aboard in situ investigation missions.

The structure of the paper is the following: Section 2 introduces the TDEM survey basic concepts (functioning principles, modelling and data interpretation). In Section 3 there is a brief overview of the most recent discoveries concerning the main issue «search for water on Mars», the space programs dealing with it and some mission scenarios well suited to a TDEM survey. In Section 4 the sounding capability, that we could expect performing a TDEM survey on Mars, is estimated for a simple case study. Some final remarks will conclude the paper.

\section{TDEM generalities}

TDEM is a controlled source, remote sensing, time domain electromagnetic (EM) method operating at the diffusion regime of EM propagation and sensitive to the conductivity gradient.

Unlike the frequency method (FDEM), it is possible to retrieve information from different depths with a single measurement (West et al., 1984; West and Macnae, 1991; Nabighian and Macnae, 1991; Zhdanov and Keller, 1994; Parasnis, 1996).

The system consists of a transmitting antenna (TX) and one (or more) receiving antenna (RX) placed on the surface with respect to a chosen configuration. For example, in the dual loop, separated and horizontal coplanar configuration, the TX and the RX are placed with the axes perpendicular to the surface. The TX is a single or multi-turn loop (Vertical Magnetic Dipole, VMD) while the RX may be a coil or a loop of wire, but a sensitive wideband magnetometer can be used as well.

The functioning principles of a TDEM survey are here briefly summarized (West and Macnae, 1991): the TX is energized with a square wave current for a time in the order of milliseconds then the current is abruptly interrupted. This step-like impulse generates a sharp magnetic field variation which, according to Faraday's law of induction, causes eddy currents in any conductive region of the medium. In their turn, such currents determine a secondary magnetic field that gradually decays in time (see fig. 1).

The RX measures the secondary magnetic field (in the case of a magnetometer) or its time derivative (i.e. the induced voltage, in the case of a coil). The signal is recorded as a function of time and processed according to proper sampling/stacking criteria. The amount of delay, strength and shape of the signal characterize the geoelectrical structure of the medium completely.

\subsection{Modelling a TDEM survey}

The main survey parameters are the distance from the source to the receiver coil, $r$, and the time of observation $t$.

Other parameters concerning the system design are the current square wave shape $I(t)$ (an impulse of amplitude $I_{0}$, and duration $T$ starting from the instant $t=0$ ) and the size of the source and receiver coils in terms of area $\left(A_{S}, A_{R}\right)$ and number of turns $\left(n_{S}, n_{R}\right)$.

The following formula expresses, in a very concise way, the dependency of the recorded measure (the transient voltage observed at the receiver) on the parameters, under the assumption that the TX and RX are sufficiently small so that the magnetic field measurement is considered as point measurement and the TX is considered a magnetic dipole:

$$
\begin{aligned}
& \left.V(t, r)\right|_{t \geq 0}=-\frac{\partial \Phi}{\partial t}=-n_{R} \mu_{0} \int_{A_{R}} \frac{\partial H_{z}(r, t)}{\partial t} d S \cong \\
& \cong-n_{R} A_{R} \mu_{0} \frac{\partial H_{z}(r, t)}{\partial t}=-M f(\sigma(\vec{p}), r, t)
\end{aligned}
$$

where $\Phi$ is the magnetic flux; $\mu_{0}$ is the vacuum permeability; $H_{z}$ is the vertical component of the magnetic field at distance $r$ from TX; $M=A_{S} A_{R} n_{S} n_{R} I_{0} ; \sigma(\vec{p})$ is the medium conductivity 


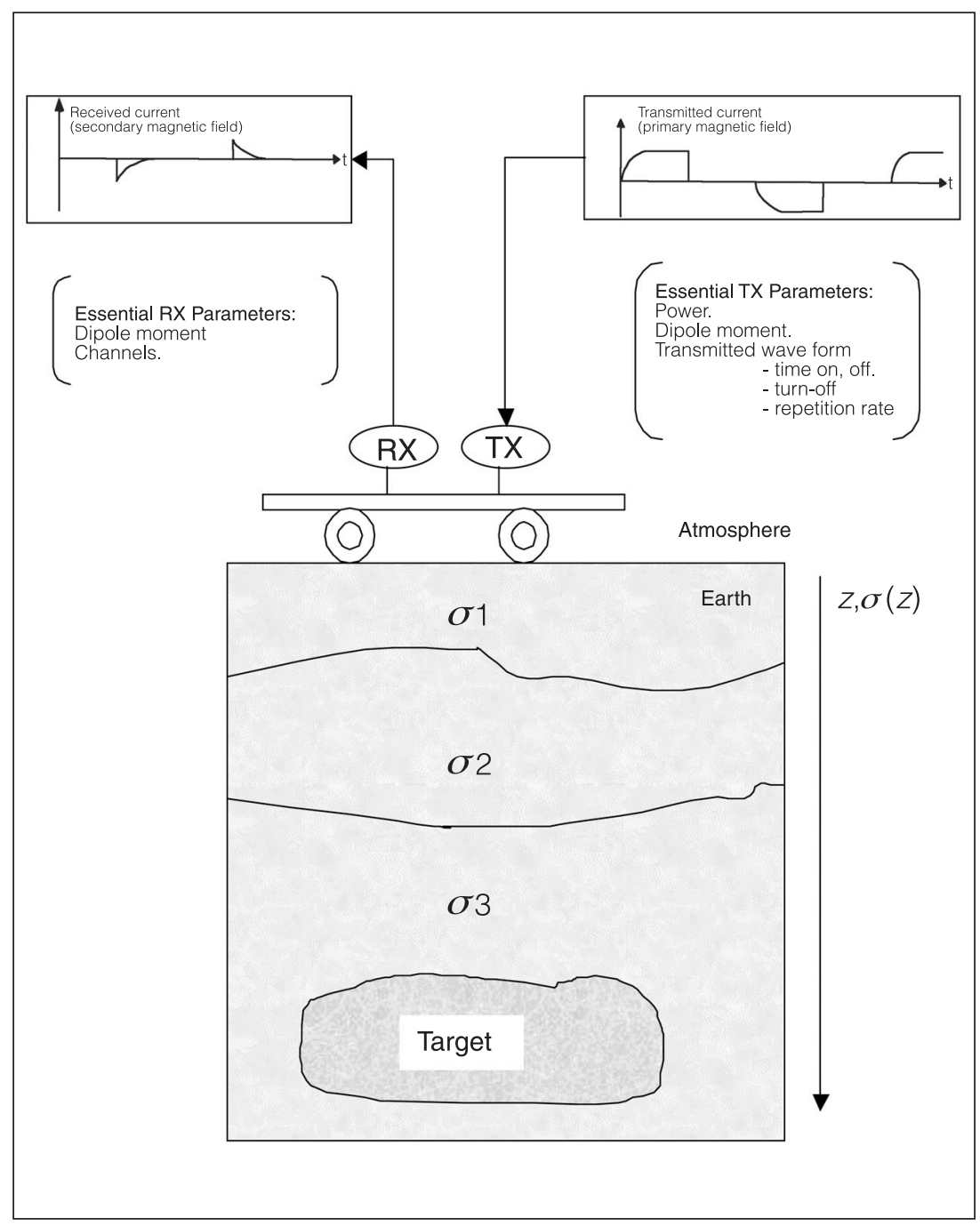

Fig. 1. TDEM experiment.

and $\vec{p}=(x, y, z)$ is the position of the conductive medium with respect to TX.

Equation (2.1) is affected by errors due to the non-homogeneities of the measurements (geometric errors) and inaccuracy of the model (e.g., deviations from constant parameters assumption). Also, it is affected by the EM background noise (Spies and Frischknecht, 1991). These are possible sources of misinterpretations and need to be considered when formulating the model, in order to assess the desired level of robustness.

The choice of the system parameters is strongly related to the required inspection depth (i.e. the scale of the survey). The multiplying factor $M$ contains the system parameters and acts as amplifier of the received signal. The dependency on the distance $r$ and time of 
observation $t$ are most important for data interpretation (geometric sounding). This fact shall be treated later on.

The medium geolectric profile $\sigma(p)$ is the unknown parameter of the experiment and it is estimated as a function of depth (laterally uniform model) or more generally as a function of the distance from the source coil (3D models). When a layered medium is assumed, the first modelling approach is computationally less demanding.

For the laterally uniform models, the parameters are the thickness and the conductivity of each layer. The rank of a model, namely the number of its layers, determines the accuracy and resolution of the subsoil mapping. Whenever the ground is approximately horizontally layered, the diffusion of the EM field has a qualitative interpretation which helps understanding of the phenomenon (Nabighian and Macnae, 1991). The EM primary field diffuses downward as a plane wave because of the high air-surface impedittivity, which implies the electric field to lie in a horizontal plane. The eddy currents are horizontal and concentric below the transmitter loop and diffuse away through the medium like smoke rings. If the ground were perfectly insulating, the waves could penetrate without attenuation, not returning back any scattered secondary field. On the contrary, in the presence of a conductive medium, the incident energy is partially absorbed and spread. This is due to the low pass filter behavior of the medium (the lower frequencies are the most penetrating). The quantity accounting for the diffusion phenomenon is the diffusion depth which relates the time $t$ (from the source impulse) to the depth of penetration $d$ and is defined as the distance over which the primary field amplitude is reduced by a factor of 1/e (Keller and Zhdanov, 1995)

$$
d=\sqrt{\frac{2 \pi t}{\sigma \mu_{0}}} .
$$

As the wave penetrates the ground, a scattered vertical magnetic field may arise from any conductive layer to the surface with a delay proportional to the depth of the layer itself. The flow of energy into the ground and back from the conductive targets (by induction) resembles the flow of energy in conventional HF radars (by reflection) so that it is also possible to give a radarlike interpretation of the above phenomenon: a conductor subjected to a time varying EM field returns back a secondary EM perturbation (i.e. the echo). The strength of the echo and the time it takes to reach the receiver depend in a very complex way on the conductivity, depth and thickness of the target, and also on the conductivity of the above layers (similar to a screen).

The EM background noise is the most important disturbing factor for any active EM sounding system. This is due to the large number of interferences which range from Ultra Low Frequency (ULF) up to Ultra High Frequency (UHF) bands. Basing on the Earth experience, the noise is originated by the contribution of two different sources: the instrumental noise, due to the TDEM equipment and the carrier, and the EM natural background noise, due to the natural magnetic field variation, to the interaction with extraneous sources (solar wind) and to the atmospheric phenomena (lightning). In order to carefully characterize the statistical model of the Martian EM background noise, it is necessary to take into account the different contribution of noise in terms of Gaussian and non-Gaussian components. This a priori knowledge is useful to maximize the signal to noise ratio (SNR), for the detection/estimation process.

The following formula expresses a possible statistical noise model:

$$
n(t)=a(t)+g(t)+c(t)
$$

$a(t)$ represents the contribute due to stationary harmonic components (micropulsations like the Earth's Schumann resonances), $g(t)$ denotes Gaussian background noise and, $c(t)$ represents randomly occurring interferences (lightning and instrument power switch-on/off). Such noise characteristics allow one to adopt the most advanced filtering and noise rejection techniques (Macnae et al., 1984; Nikias and Mendel, 1993).

Once the data have been recorded and filtered, they have to be interpreted. The interpretation relies on the solution of the forward and inverse 
problem. The inverse problem says: «Given an EM transient signal, determine the geo-electric structure of the medium that generated it» (Zhdanov and Keller, 1994).

It consists of estimating the geoelectric structure of the Earth that generates the recorded signal.

The dual problem is the forward problem which says: «Given a geo-electric structure of the Earth, determine the EM transient signal at the receiver» (Zhdanov and Keller, 1994).

The forward problem must be solved in order to evaluate the response of the model for a given parameter setting (in the chosen model parametric space). The inversion problem consists of finding the model that best fits the recorded data. Both problems are represented in the block diagram of fig. 2, where the TDEM experiment has been modelled in accordance with a signal processing point of view.

In the laterally uniform model, the main advantage is that data inversion is unique (Zhdanov and Keller, 1994). If needed, it could be even possible to provide a more detailed subsoil mapping solution by processing the same data with reference to a 3D model, but remembering the intrinsic computational complexity in managing the data inversion in such a context. Moreover, in this, the interpretation becomes ambiguous and the problem of the data inversion is said to be «ill posed».

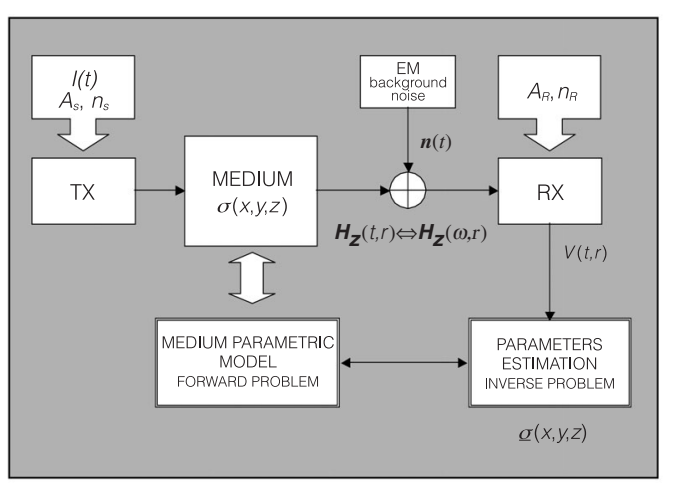

Fig. 2. Block diagram of the TDEM measurement process.
An alternative approach is based on probabilistic tomography (Patella, 1997; Mauriello and Patella 1999). The data interpretation is no longer bounded to any preconceived subsoil model (model free approach) and the results have to be interpreted in a probabilistic sense. It has worked successfully in the mapping of complex geoelectric profiles as well as in the localization of water. Thanks to a lower computational cost (with respect to the canonical 3D modelling) and potentially no limit to the range of application (TDEM, IP, Magnetotelluric, etc.), it represents a valid alternative to the more traditional approaches, and also for subsequent refinement and result validation.

\section{Insights into water on Mars and some future mission scenarios}

Many hypotheses about the presence of water on Mars have been formulated thanks to the scientific data provided by some past and more recent missions, like the Viking (1975), the Pathfinder (1996) and the Mars Global Surveyor (1996) and more recently the Mars Odyssey (2001). An outline of the results is briefly reported here (Briggs, 2000; Heggy et al., 2001):

The Martian atmosphere - Martian atmosphere is extremely dry and water exists as ice or vapor in trace quantities $(0.03 \%)$.

The Martian surface - A dust layer of a mixture of conducting (iron oxides, hematite, etc.) and less conducting material (silicates and basaltic rocks) covers almost the whole planet. Water exists as ice at high latitudes (on the poles) from which it sublimates during the Martian summer.

The Martian subsurface - Due to the evidence of superficial water-related processes (crustal erosions and deep valleys), water has been supposed to exist even in the subsurface.

The presence of water on the Martian surface is theoretically possible as a temporary phenomenon, at certain latitudes and times 
(due to a heating of the crust or to a superficial geothermal activity), and the major expectations rely upon the subsoil exploration, where very likely it should exist in the form of a mixture of ground and ice, similar to the terrestrial permafrost.

\subsection{Mission scenarios}

The different exploration programs developed by the major space agencies (NASA, ESA, ASI, ...) in the latest few years have agreed on splitting the Martian exploration into two distinct phases: a first exploration phase including a large scale mapping of the Martian surface from satellites, followed by a second phase of in situ investigations.

At present, there exists a great expectation on the Martian in situ exploration and a large variety of exploration scenarios have been conceived, from the missions consisting of a single rover or a lander equipped with a heavy package of scientific instruments (the single station approach), to more ambitious missions (multi-station approach) consisting of a set of rovers, landers and robots (Brack et al., 1999; Heggy et al., 2001). Although single as well as multi-station scenarios are well suited to a TDEM experiment, the multi-station approach (see fig. 3) would offer the best platform for a geophysical distributed TDEM experiment, that means: a single transmitter and a certain number of receivers (one or $\mathrm{N}$ ) which could be housed aboard each carrier fixed or moving (lander, rover, robots) with respect to a chosen configuration. A distributed TDEM experiment will allow: 1) simultaneous measurements with respect to a proper geometric sounding approach (time-space correlated observations); 2) a higher resolution in the measurement (qualitative improvement), by clustering in groups a certain number of stations; and finally, 3) exploration of a larger area recording a significant amount of data for the fixed slice of time available (quantitative improvement). Other advantages of the distributed configuration,

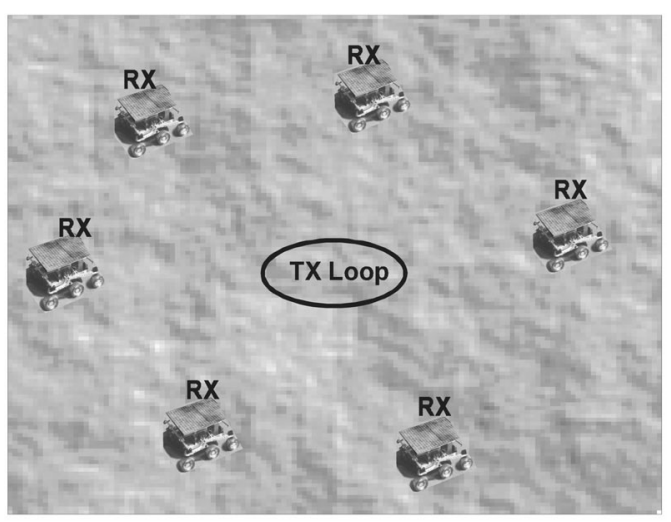

Fig. 3. Rover distributed configuration.

Table I. Mission scenarios for a TDEM survey.

\begin{tabular}{|c|c|c|c|}
\hline & & Single station & Multi-stations \\
\hline \multicolumn{2}{|c|}{ General features } & $\begin{array}{l}\text { Multi-purpose, heavy } \\
\text { packaged, high cost and } \\
\text { mass. }\end{array}$ & Special-purpose, low cost and mass \\
\hline \multicolumn{2}{|c|}{ Survey scale } & $\begin{array}{l}\text { Local survey: restricted area } \\
\text { of exploration. }\end{array}$ & $\begin{array}{l}\text { Global survey: larger area of } \\
\text { exploration; geometric sounding. }\end{array}$ \\
\hline \multirow[t]{2}{*}{$\begin{array}{l}\text { Survey } \\
\text { scenarios }\end{array}$} & Fixed & $\begin{array}{l}\text { Lander: traditional single } \\
\text { survey. }\end{array}$ & $\begin{array}{l}\text { Multi-Lander: } \\
\text { Simultaneous multi-sites survey. }\end{array}$ \\
\hline & Mobile & $\begin{array}{l}\text { Rover (MSR-NASA): } \\
\text { multi-survey approach. }\end{array}$ & $\begin{array}{l}\text { Micro-robots (the next future) } \\
\text { The same as above plus variable, task } \\
\text { adaptive survey configuration. }\end{array}$ \\
\hline \multicolumn{2}{|c|}{$\begin{array}{l}\text { TDEM system } \\
\text { configuration }\end{array}$} & $\begin{array}{l}\text { Coincident or concentric } \\
\text { transmitter-receiver }\end{array}$ & $\begin{array}{l}\text { Separated transmitter-receiver(s); (1:1) } \\
\text { or }(1: \mathrm{N}) .\end{array}$ \\
\hline
\end{tabular}


with respect to the single station approach, is the possibility to employ a less expensive carrier (smaller and even technologically less complex), experiment flexibility (possibility to perform multi-sites survey by means of the system reconfiguration facility) and increased reliability (in term of probability of mission completion). In table I, some of the mission scenarios are classified with respect to some very general features, pointing out the single capabilities and facilities for a TDEM survey.

\section{TDEM experiment design and expected performance in a planetary mission context: a case study}

Within a planetary mission context, the payload scientific experiments are subjected to more or less evident limitations. The major constraints deal with mass, dimension, power, mobility and other key resources depending also on the type of mission. These limitations together with mentioned EM background noise and first layer screening effect, compromise the system performance.

The TDEM performance is the combination of two quantities, the depth of investigation plus the resolution (Spies and Frischknecht, 1991). The former is the maximum depth of penetration (generated by the source impulse), scattering a detectable signal back to the surface (echo to the receiver), for a given maximum threshold of sensitivity (i.e. receiver sensitivity). The latter represents the ability to detect the conductivity and thickness of a layer in the subsoil.

System sounding capabilities are evaluated here for a simple case study wherein a saltwater deposit lying at a certain depth beneath the surface is the target of the investigation. The following assumptions hold:
TDEM system

- Separated transmitter-receiver configuration (1:1). TX and RX are coils.

- Ideal receiver.

- Ideal square wave (step function) current source.

Martian subsoil and EM background noise

- Laterally uniform subsoil and a thin sheet model for target resolution measurement.

- EM background noise (after filtering and stacking) under the threshold of sensitivity.

System and model parameters are shown respectively in tables II and III. When needed, a different range of values will be specified. The time $t$ and the spacing $r$ are the free parameters of the study.

\subsection{Screening effect and depth of investigation}

A very conductive layer causes the EM field to loose a large amount of energy (the Ohmeffect), remaining confined within the shallow subsurface. At the same time, the penetration velocity (depending on the conductivity) decreases so that the EM wave reaches any potential target later and with not enough energy to scatter back a detectable signal. This effect is called the

Table II. System parameter settings.

\begin{tabular}{ll}
\hline \hline \multicolumn{1}{c}{ System parameters } & \multicolumn{1}{c}{ Values } \\
\hline$A_{S}, A_{R}$ & $1 \mathrm{~m}^{2}$ \\
$n_{S}, n_{R}$ & 112 turns \\
$I_{0}$ & $1 \mathrm{~A}$ \\
Dipole moment $M$ & $12544 \mathrm{~A} \mathrm{~m}^{4}$ \\
$\left(n_{S} n_{R} A_{s} A_{R} I_{0}\right)$ & \\
Threshold of sensitivity & $\propto \mu \mathrm{V}$ \\
\hline
\end{tabular}

Table III. Conductivity profile range of values.

\begin{tabular}{ccc}
\hline \hline Martian subsoil & $\begin{array}{c}\text { Conductivity expected values } \\
\Omega^{-1} \mathrm{~m}^{-1}\end{array}$ & $\begin{array}{c}\text { Thickness } \\
(\mathrm{m})\end{array}$ \\
\hline First layer & 0.001 silicates $\div 0.1$ iron-oxides & $1 \div 100$ \\
Target & Water (saline) 4 & 10 \\
\hline
\end{tabular}


screening effect and it represents the most serious limitation for the detection of any buried target. A rough estimation of the screening effect could be obtained by considering the attenuation of the plane wave diffusing downward an uniform medium of conductivity $\sigma$. In this case, the voltage time response at the receiver (see eq. (2.1) ) can be analytically expressed as follows (Zhdanov and Keller, 1994):

$$
\begin{gathered}
\left.V(t, r)\right|_{t \geq 0}= \\
=M \frac{9}{2 \pi \sigma r^{5}}\left[\operatorname{erf}(u)-\sqrt{2 / \pi} e^{-\frac{u^{2}}{2}} u\left(1+\frac{u^{2}}{3}+\frac{u^{4}}{9}\right)\right] \\
u=\frac{2 \pi r}{d} ; \operatorname{erf}(u) \text { error function. }
\end{gathered}
$$

For simplicity, (4.1) may be approximated with the following asymptotic expression holding at late time:

$$
\left.\lim _{t>>0} V(t, r)\right|_{t \geq 0} \cong M \frac{4 \pi \sigma^{3 / 2} \mu_{0}^{5 / 2}}{t^{5 / 2}}
$$

The time $t$ can be made explicit in (4.2) and substituted in eq. (2.2) to obtain the expression of the maximum depth of investigation as a

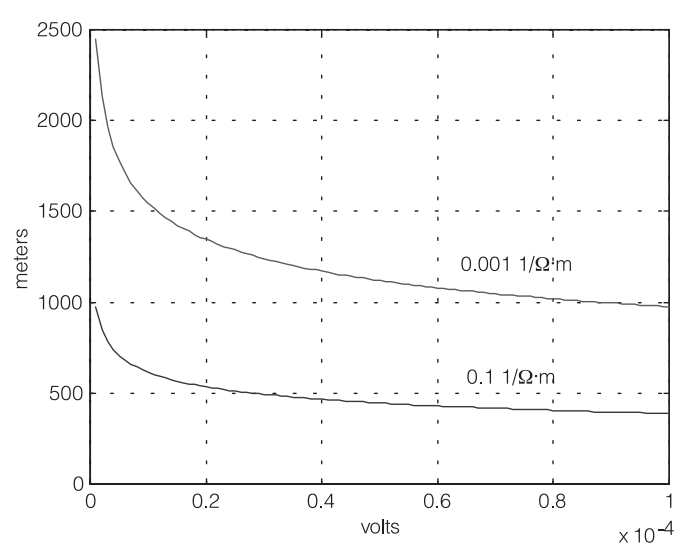

Fig. 4. Diffusion depth of a transient EM plane wave versus threshold of sensitivity. function of the threshold of sensitivity, the medium conductivity and the gain $M$ (Spies and Frischknecht, 1991)

$$
d=(4 \pi)^{7 / 10}\left(\frac{M}{\sigma V_{t h}}\right)^{1 / 5} .
$$

The expression gives an approximated information on the maximum depth of investigation. Figure 4 shows the results obtained for the two first layer conductivity values assumed in table III, confirming how a more conductive first layer limits the penetration.

\subsection{Target resolution}

When the conductivity difference between a conductive layer and the surrounding medium is more than 100 , then there exists a well suited approximation for the analytical evaluation of a TDEM experiment: the thin sheet model (Nabighian and Macnae, 1991; Zhdanov and Keller, 1994).

Herein, the medium is approximated by an insulator while the target layer is modeled as a thin sheet lying at a certain depth $D$ from the surface. Its conductance is the product of the conductivity $\sigma_{T}$ for the thickness $T$. The voltage time response at the receiver (see eq. (2.1) ) can be analytically expressed as

$$
\begin{gathered}
\left.V_{\text {coil }}(t, r)\right|_{t>0}=-\frac{M}{\pi S} \frac{u(t)\left[-9 r^{2}+24 u(t)^{2}\right]}{\left[r^{2}+4 u(t)^{2}\right]^{7 / 2}} \\
u(t)=D+\frac{t}{\mu_{0} S} ; S=\sigma_{T} T .
\end{gathered}
$$

This model is mostly useful to highlight the echo scattering and the importance of relating together the recording time, the spacing between the coils and the depth of the target.

Let us assume a target of conductance $S=4 \times 10 \Omega^{-1}$ (salt water), a fixed time $t=$ $=0.01 \mathrm{~ms}$ and evaluate the expression (4.4) for different values of $D$. The results (see fig. 5) show there exists an optimal receiver spacing in order to detect the strongest signal, once the 


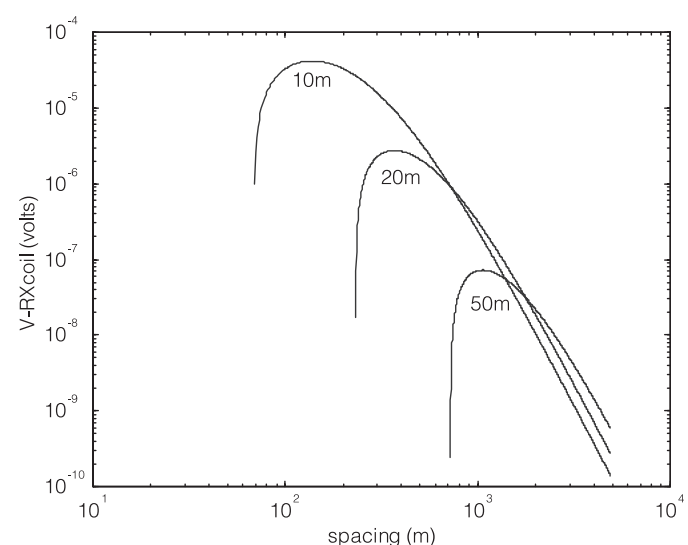

Fig. 5. Voltage response at the receiver for different target depth and fixed time window $=0.01 \mathrm{~ms}$.

recording time window has been selected. By definition, a time window $T_{W}$ is the time interval within which we expect to observe the signal scattered by a conductor lying at a certain depth. The dependency of the time window $T_{W}$ on the depth of penetration $D$ and spacing $r$ between the coils is a strong result of the experimental evidence. The following simple linear equation, which relates the optimal spacing $r$, the depth of the target $D$ and the $T_{W}$ (see also fig. 6), holds:

$$
\text { optimal_spacing } \cong 0.4 D+c T_{W}
$$

where the constant $c$ depends on the time window.

This expression resumes the properties of the geometric sounding. It says that it is possible to control the depth of investigation by simply moving the receiver coil and setting the time of observation to a certain time window so as to retrieve the maximum response signal from the target. This way, just repeating the same measurements at different locations, it is possible to achieve an accurate 2D map of the subsoil conductivity.

Figure 7 shows the maximum voltage at the receiver as a function of the target depth and for different time windows. Of course the spacing must be adjusted as a function of depth. Given the threshold of sensitivity of the receiver, it is possible to assess the limit of investigations in terms of maximum signal detectable and therefore maximum reachable target depth. For instance, let us assume a $1 \mu \mathrm{V}$ threshold, the maximum detectable target should lie at almost $20 \mathrm{~m}$ (time window $=0.01 \mathrm{~ms}$ ), and if the threshold was 0.01 $\mu \mathrm{V}$ it could be possible to detect the target even at $100 \mathrm{~m}$ deep.

Due to the model simplification (absence of screening effect) and noise free experiment assumption, the results must be considered the upper limit for the system performance. Relaxing

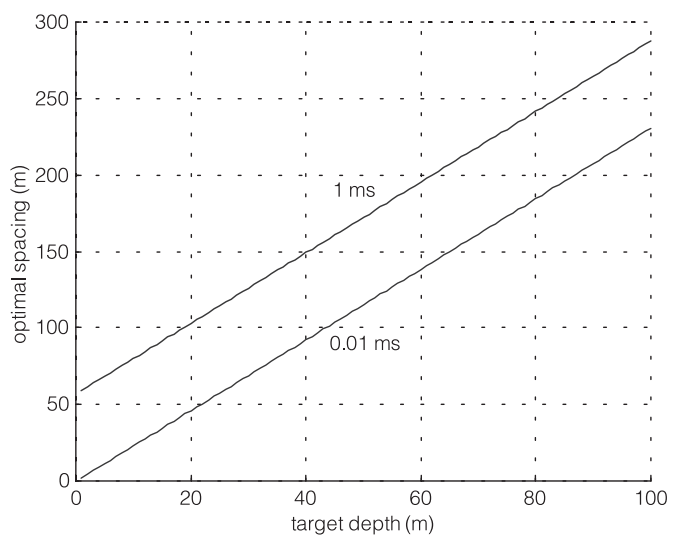

Fig. 6. TX-RX optimal spacing $r$ in function of the target depth.

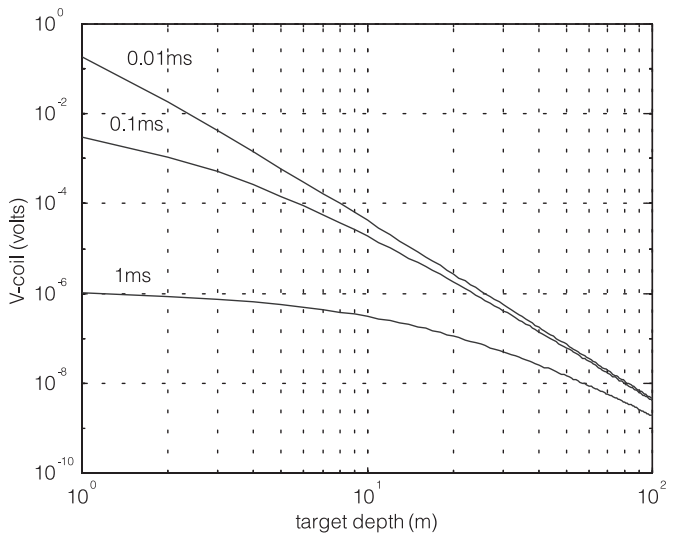

Fig. 7. Maximum voltage response at the receiver in function of target depth, for different time windows. 
the assumptions means to consider at least a twolayered model for the subsoil, to add noise to the signal and to model the dynamical behavior of the TX and RX with the respective transfer functions. In so doing, a significant spreading and lowering for the time response is expected, the amount of attenuation and delay depending on the first layer conductivity. The dynamics of TX and RX, which depend on the number of turns and area, could affect the selection of the recording time window of the experiment. Namely, the experiment requirements consist of: 1) a very fast transient response for the TX (the loop working better than a coil), so that the primary field does not interfere directly (by TX-RX mutual coupling) with the secondary field, and 2) a RX cut-off frequency not too low (i.e. $>1000 \mathrm{~Hz}$ ) in order to achieve the desired level of resolution. Advanced design solutions exist in order to counteract the above potential limitations. Some of these have already been implemented in commercial systems. For instance, a very early recording time is feasible by spacing opportunely TX and RX or alternatively arranging them in a null-coupled configuration (zero mutual impedance).

\section{Conclusions}

The paper has investigated the feasibility of a TDEM system for Martian subsurface geophysical exploration. The operational environment has been defined and some of the potential obstacles, either of technical or environmental nature, have been highlighted. A simple case study showed how mission constraints could limit the system sounding capability. In such a context, even a few tens of meters of penetration should be a quite remarkable result. This is quite satisfactory for the exobiology research program, where the role of a TDEM system could be to target a drill to a suitable site for sampling activity. On the contrary, greater depths are required for water search task. Indeed, this kind of problem is usually encountered whenever a methodology, well assessed on the Earth, must be adapted for a space application. Thus, a very special attention in designing the system in each single part would be required: the transmitter should generate the greatest source impulse with respect to the low maximum peak of current available, the receiver has to be sensitive to very low signals, the EM noise characterization and data acquisition strategy will be necessary in order to improve the $\mathrm{S} / \mathrm{N}$ ratio. Altogether with these design recipes, it is worthwhile also thinking of a drastically different approach to the TDEM experiment. For instance, just considering the more futuristic mission scenarios, let us suppose having a network of carriers available. The TDEM survey might be arranged as follows: a rover could unroll and stand a wire on the surface where the survey is planned and, at the same time, a set of receivers could be arranged in a networklike configuration on the surface. This way, the area of the transmitter would be increased with a resulting advantage in a larger energizing moment, and the data could be acquired in different places, simultaneously, also increasing the $\mathrm{S} / \mathrm{N}$ ratio by means of the multisensor data fusion. This latter approach would represent the common framework for many of the in situ scientific experiments.

A package of instruments, cooperating for the same objective, represents the most promising solution in a such extreme environment. Just referring to the Earth experience, there exist many examples where the combination of two or more sounding methodologies have increased the overall performances (Meju, 1996). This approach is also recommended to obtain the best opportunities of employment or/and merging of the TDEM with other sounding methods and represents, in our opinion, together with the distributed configuration approach, the most promising way to successfully perform a TDEM geophysical survey on Mars.

\section{Acknowledgements}

The presented study has been conducted in the context of TESEO, a project supported by the Italian Space Agency (ASI), in collaboration with CNR-ISSIA, DIBE-University of Genoa, DSF-University «Federico II of Naples», IRSPSUniversity of Pesaro and Officine Galileo. We are also mostly grateful to Prof. Domenico Patella for fruitful discussions on the subject. 


\section{REFERENCES}

Brack, A., P. Clancy, B. FitTon, B. Hoffman, G. HoRnECK, G. Kurat, J. Maxwell, G. Ori, C. Pillinger, F. RAulin, N. Thomas and F. Westall (1999): An integrated exobiology package for the search for life on Mars, Adv. Space. Res. 23 (2), 301-308.

Briggs, G. (2000): Follow the water, Meteoritics Planet. Sci., 35 (5), 892-893.

Heggy, E., P. Paillou, G. Ruffie, J.M. Malezieux, F. COSTARD and G. GRANDJEAN (2001): On water detection in the martian subsurface using sounding radar, Icarus, 154 (2), 244-257.

MaCnAE, J.C., Y. LAMONTAGNE and F.G. West (1984): Noise processing technique for time-domain EM systems, Geophysics, 49 (7), 934-948

Mauriello, P and D. Patella (1999): Principles of probability tomography for natural-source electromagnetic induction field, Geophysics, 64 (5), 1403-1417.

MEJU, M.A. (1996): Joint inversion of TDEM and distorted MT soundings: some effective pratical considerations, Geophysics, 61 (1), 56-65.

NABIGHIAN, N. and J.C. MACNAE (1991): TEM prospecting methods, in Electromagnetic Methods in Applied
Geophysics, edited by N. NABIGHIAN (SEG), vol. 2 , 427-479.

NIKIAS, C.L. and J.M. MENDEL (1993): Signal processing with higher-order spectra, IEEE Signal Process. Mag., 10, 10-37.

PARASNIS, D.S. (1996): Principles of Applied Geophysics (Kluwer Academic Publishers, Dordrecht), pp. 456.

PATElla, D. (1997): Self-potential global tomography including topographic effects, Geophys. Prospect., $\mathbf{4 5}$, 843-863.

SPIES, B.R. and F.C. FRISCHKNECHT (1991): Electromagnetic sounding, in Electromagnetic Methods in Applied Geophysics, edited by N. NABIGHIAN (SEG), vol. 2, 285-386.

West, F.G. and J.C. Macnae (1991): Physics of the electromagnetic induction exploration methods, in Electromagnetic Methods in Applied Geophysics, edited by N. NABIGHIAN (SEG) vol. 2, 5-46.

WeSt, F.G., J.C. MACNAE and Y. LAMONTAGne (1984): A TDEM system measuring the step response of the ground, Geophysics, 49 (7), 1010-1026.

ZHDANOV, M.S. and G.V. KELLER (1994): The Geoelectrical Methods in Geophysical Exploration (Elsevier, Amsterdam-New York-Tokyo), vol. 31, pp. 873 